\title{
ON CONTINUATION OF REGULAR SOLUTIONS OF LINEAR PARTIAL DIFFERENTIAL EQUATIONS
}

\author{
AKIRA KANEKO \\ Department of Mathematics, College of General Education, University of Tokyo \\ Tokyo, Japan
}

In this talk we survey results on continuation of solutions of linear partial differential equations. We can classify this problem, roughly speaking, into three categories as follows:

(1) continuation to a large set (e. g. across a hypersurface; problem of hyperbolicity);

(2a) continuation to a small set (problem of removable singularity), with growth condition on the solution;

(2b) as above, but without growth condition.

Our research mainly concerns problems of type (2b). As a survey, however, we shall start with the general story. As is well known, the origin of this study lies in the problem of removable singularities in the theory of functions of a single complex variable (category (2a) in this classification). Already as early as 1851, Riemann [1] had pointed out that an isolated singularity of a holomorphic function is removable if the function is bounded around the singularity. Later, in 1906, Hartogs [1] proved that in the case of holomorphic functions of several variables any convex compact set is removable, irrespective of the boundedness of the function. After that, studies extending these results were made by researchers in complex analysis. Among them we mention that Hartogs' theorem was generalized by these people to any pair $K \subset U$, where $K$ is the part of a convex compact set lying in a half space and $U$ is a neighborhood (although this form of the assertion also bears Hartogs' name nowadays). See Struppa [1] for a detailed history of these.

In 1952 Bochner [1] gave a new proof of Hartogs' theorem employing the notion of an overdetermined system of differential equations. This initiated an abundant study of the problem of continuation of solutions in the theory of partial 
differential equations. In particular, a satisfactory generalization of the Hartogs theorem was given by Ehrenpreis [1], Malgrange [2], Palamodov [1], Komatsu [1]. We present here a typical result:

Theorem A. Let $K$ be a convex compact subset of $\mathbb{R}^{n}$ and let $U$ be an open neighborhood. Then for a matrix $P(D)$ of linear partial differential operators with constant coefficients, the following are equivalent:

1) Every hyperfunction solution $u$ of the system $P(D) u=0$ defined in $U \backslash K$ can be continued to $U$.

2) $\operatorname{Ext}^{1}\left(\operatorname{Coker}^{t} P, \mathcal{P}\right)=0$.

Here $\mathcal{P}$ denotes the ring of polynomials in $\zeta$ (or $D)$ and ${ }^{t} P$ denotes the transposed matrix of $P(\zeta)$. The continuation is unique if and only if $\operatorname{Hom}\left(\operatorname{Coker}^{t} P, \mathcal{P}\right)=0$.

We emphasize that we took hyperfunction solutions here because of the simplicity of the corresponding result. If we take distribution solutions, we have to replace $K$ by an open set, as in Malgrange, or introduce a notion related to an inductive limit around the set $K$, as in Palamodov. This annoyance comes from the non-extendibility of general distributions from an open set, and arises only when $\operatorname{Hom}\left(\operatorname{Coker}^{t} P, \mathcal{P}\right) \neq 0$. The matter is similar for infinitely differentiable solutions, but then an additional restriction that the interior of $K$ must be non-void is required, as we shall see below. In any case, note that the above theorem concerns systems. Since in this talk we mainly treat single equations, we will not give definitions for these notions concerning systems. Notice only that for a single operator $P$, Coker ${ }^{t} P=\mathcal{P} /{ }^{t} P \mathcal{P}, \operatorname{Ext}^{1}\left(\operatorname{Coker}^{t} P, \mathcal{P}\right) \cong \mathcal{P} / P \mathcal{P}$, and this latter is 0 if and only if $P$ is non-zero constant. Also note that $\operatorname{Hom}\left(\operatorname{Coker}^{t} P, \mathcal{P}\right)=0$ if and only if $P=0$. These facts have already been observed in Ehrenpreis [1].

If we consider a sufficiently regular solution with a thin singularity $K$, then taking the equation $D_{1} u=0$ as an example, we can easily see that the situation of continuability of solutions will differ greatly because of the "unique continuation property". Grushin [2] gave the following wide class of single equations which admit this property:

TheOREM B. Let $P(D)$ be a single linear partial differential operator with constant coefficients. Assume that every irreducible factor of $P$ has a simple characteristic direction. Then every isolated singularity of infinitely differentiable solutions of $P(D) u=0$ is removable.

Our study was to clarify this phenomenon from as general a viewpoint as possible. This is a particular case of the continuation problem belonging to category $(2 b)$ in our classification at the beginning. In the sequel we shall restrict our talk mainly to continuation of real-analytic solutions. (Some of the results are extended to quasi-analytic solutions. See Abramczuk [1].) We list here the main results among them: 
Theorem C (Kaneko [1], [6]). Let $K$ be a convex compact set and let $U$ be its neighborhood. For a linear partial differential operator $P$ with constant coefficients, the following are equivalent:

1) Every real-analytic solution of $P(D) u=0$ defined on $U \backslash K$ can be continued to $U$.

2) $P$ has no elliptic irreducible factor.

There is a version for systems (Kaneko [1], II). It suffices to replace $P$ by $\operatorname{Ext}^{1}\left(\operatorname{Coker}^{t} P, \mathcal{P}\right)$ in 2$)$. To assure the real analyticity of the continued solution, however, we must assume $\operatorname{Hom}\left(\operatorname{Coker}^{t} P, \mathcal{P}\right)=0$, which is automatic for single operators. The condition of convexity of $K$ can be relaxed to the connectedness of $\mathbb{R}^{n} \backslash K$ (Kaneko [3]). The result contains as a particular case Severi's generalization of Hartog's theorem to real-analytic functions which are holomorphic in some of the variables (Severi [1]).

In order to state a variant of non-compact type $K$ we shall say in general that $K \subset U$ is a Hartogs pair if $K$ is the intersection of a convex compact set with the half-space $x_{n}<0$, and $U$ is an open neighborhood. Actually, the convexity of $K$ can be replaced by the connectedness of $\left\{x_{n}<0\right\} \backslash K$ as above. We shall give an explanation, however, for the simplest situation.

Theorem D (Kaneko [14]). The following are equivalent:

1) The continuation of real-analytic solutions holds for every Hartogs pair.

2) For any $A, a>0$ we can find $B \geq A, b<a, \delta>0$ such that the following Phragmén-Lindelöf type principle holds for global holomorphic functions on every irreducible component $N_{\lambda}$ of the variety $N(P):=\left\{\zeta \in \mathbb{C}^{n} ; P(\zeta)=0\right\}$ : If $F(\zeta)$ satisfies, for any $\varepsilon>0$,

$$
|F(\zeta)| \leq C_{\varepsilon} \exp \left(\varepsilon|\zeta|+A\left|\operatorname{Im} \zeta^{\prime}\right|+a\left(\operatorname{Im} \zeta_{n}\right)_{-}\right) \quad \text { for } \forall \zeta \in N_{\lambda},
$$

and, for any $\varepsilon>0$,

$(\beta)|F(\zeta)| \leq C_{\varepsilon} \exp \left(\varepsilon|\zeta|+A\left|\operatorname{Im} \zeta^{\prime}\right|+b|\operatorname{Im} \zeta|\right) \quad$ on $N_{\lambda} \cap\{|\operatorname{Im} \zeta| \leq \delta(|\operatorname{Re} \zeta|+1)\}$, then it satisfies, for any $\varepsilon>0$,

$$
|F(\zeta)| \leq C_{\varepsilon} \exp \left(\varepsilon|\zeta|+B\left|\operatorname{Im} \zeta^{\prime}\right|+b|\operatorname{Im} \zeta|\right), \quad \text { for } \forall \zeta \in N_{\lambda} .
$$

Here $(t)_{-}:=\max \{-t, 0\}$ and $\zeta^{\prime}=\left(\zeta_{2}, \ldots, \zeta_{n-1}\right)$.

The above presentation imitates Hörmander's work [1] on the global solvability of real-analytic solutions, and was given rather recently. Our first works were on concrete sufficient conditions (Kaneko [2]). We give them here in a later-improved form.

THEOREM E. The continuation of real-analytic solutions for any Hartogs pair holds if every irreducible factor $Q$ satisfies one of the following:

1) There exists a sequence of directions $\vartheta_{k}$ tending to $d x_{n}$ such that $Q$ is hyperbolic in every direction $\vartheta_{k}$. 
2) $Q(D)$ has the principal part $Q_{\mu}(D)$ which does not contain $D_{n}$ and which is elliptic with respect to the variables actually contained in $Q_{\mu}(D)$.

3) $Q(D)$ is of second order and has real principal part independent of $D_{n}$.

4) The operator $Q(D)$ itself does not contain $D_{n}$.

Hyperbolicity is here understood in the weakest sense, i.e. that for the principal part. The following theorem says that hyperbolic operators are only generic ones in this context:

THEOREM F. If $d x_{n}$ is a non-characteristic direction of $P(D)$ and if the extension of real-analytic solutions holds for a Hartogs pair $K \subset U$ such that $\bar{K} \cap\left\{x_{n}=0\right\}$ has a non-void interior as a set in $\mathbb{R}^{n-1}$, then $P(D)$ is hyperbolic in the direction $d x_{n}$.

The heat equation $D_{1}^{2}+\ldots+D_{n-1}^{2}+i D_{n}$ is, however, a more interesting example of the above theorem. The concrete meaning of our abstract criterion in Theorem D for such degenerate cases is not yet very clear. It is an interesting, open question if it depends only on the principal part or not.

For the heat equation and the time-evoluting direction we can extend this result to equations with real-analytic coefficients employing the analytic semigroup theory. It is very plausible that the same is true in the opposite time direction, too, or for Schrödinger type equations. But we have no idea of tools for these for the moment.

If we consider a Hartogs pair with a thin set $K$, we can considerably enlarge the class of operators admitting the continuation of solutions:

Theorem G. Let $K$ be contained in the hyperplane $x_{1}=0$ which is noncharacteristic with respect to $P(D)$. Assume that every root $\tau\left(\zeta^{\prime}\right)$ of $P\left(\tau, \zeta^{\prime}\right)=0$, where $\zeta^{\prime}=\left(\zeta_{2}, \ldots, \zeta_{n}\right)$, satisfies the estimate

$$
\left|\operatorname{Im} \tau\left(\zeta^{\prime}\right)\right| \leq \varepsilon\left|\zeta^{\prime}\right|+b\left|\operatorname{Im} \zeta_{n}\right|+C_{\zeta_{2}, \ldots, \zeta_{n-1}, \varepsilon} .
$$

Then every real-analytic solution of $P(D) u=0$ in $U \backslash K$ can be continued to $U$.

This theorem, first proved by Fourier analysis, lies halfway to micro-local analysis. It was later reproved by micro-local analysis based on the following result on the propagation of regularity along the boundary and the Kashiwara-Kawai theorem of Holmgren type, which replaces the Phragmén-Lindelöf principle in Fourier analysis:

TheOrem H (Kaneko [9]). Assume that $P(D)$ satisfies the same condition as in the above theorem. Then the boundary values $u_{k}\left(x^{\prime}\right)=\left.D_{1}^{k} u\right|_{x_{1} \rightarrow \pm 0}, k=$ $0, \ldots, m-1$, of every real-analytic local solution $u$ of $P(D) u=0$ defined on $\pm x_{1}>0$ satisfy the following phenomenon of propagation of micro-analyticity: The set

$$
\bigcup_{k=0}^{m-1} \text { S.S. } u_{k}\left(x^{\prime}\right) \cap\left\{x_{n}=\text { const }\right\} \times\left\{ \pm d x_{n}\right\}
$$

cannot be compact. 
Once the micro-local approach is adopted, it is straightforward to generalize the problem to equations with variable coefficients. Actually the following results were given even earlier than the above somewhat delicate result:

Definition I. Consider the set of points $\left(x, \xi^{\prime} d x^{\prime}\right) \in\left\{ \pm x_{1}>0\right\} \times S^{n-2}$ such that the characteristic equation $P_{m}\left(x, \zeta_{1}, \xi^{\prime}\right)=0$ for $\zeta_{1}$ has at least one root with positive (resp. negative) imaginary part. Take its closure in $\mathbb{R}^{n} \times S^{n-2}$ and restrict it to $S=\left\{x_{1}=0\right\}$. We let $V_{S, A}^{+}(P)$ (resp. $\left.V_{S, A}^{-}(P)\right)$ denote the set of points $\left(x^{\prime}, \xi^{\prime} d x^{\prime}\right) \in \mathbb{R}^{n-1} \times S^{n-2}$ obtained in this way.

TheOREM J. Let $u$ be a real-analytic solution of $P u=0$ defined on $\pm x_{1}>0$. Then its boundary values at $x_{1} \rightarrow \pm 0$ have S.S. contained in $V_{S, A}^{ \pm}(P)$.

Corollary K. Let $K=\left\{x_{n} \geq \varphi\left(x^{\prime}\right)\right\} \cap\left\{x_{1}=0\right\}$, where $\varphi$ is a function of class $C^{1}$ such that $\varphi(0)=0, \nabla \varphi(0)=0$. If either of $\left(0, \pm d x_{n}\right)$ is outside the set $V_{S, A}(P):=V_{S, A}^{+} \cup V_{S, A}^{-}$, then every real-analytic solution of $P u=0$ defined in $a$ neighborhood of the origin outside $K$ can be continued as a hyperfunction solution to a whole neighborhood of the origin.

Note that without the assumption of "convexity" for $K$ we need not have the propagation of real analyticity, hence the continued solution need not be real-analytic. This result covers the generic case. Some more delicate cases are treated by Schapira [1], [2], [3], Kataoka [1], Kaneko [7], Ôaku [2], Zampieri [1], Uchida [1], Uchida \& Zampieri [1] etc. and are still in progress.

Changing the viewpoint, we try to study what kind of singularities are actually presented by real-analytic solutions. For this purpose it is necessary to consider singularities of minimal dimension because those of higher dimension may be as complicated as will be by means of superposition.

TheOREM L. Let $r_{S}(P)$ denote the minimal value of the codimension in $S^{n-2}$ of concentric subspheres contained in the generic fiber of $V_{S, A}(P)$. Suppose that there exists a real-analytic solution $u$ to the equation $P(x, D) u=0$ such that $u$ is defined outside $C$ but cannot be continued to a neighborhood of $C$, even as a hyperfunction solution. Then the conormals of $C$ must be contained in $V_{S, A}(P)$. (We call such $C$ weakly timelike.) Hence in particular $\operatorname{dim} C \geq r_{S}(P)$. Further, if $\operatorname{dim} C=r_{S}(P)$, it follows that $C$ is a real-analytic submanifold.

The assertion about dimension is a direct consequence of the preceding theorem. The last statement on the analyticity follows from the following generalization of a classical result of Hartogs:

LEMmA M. Let $F(z, \tau)$ be a function holomorphic on a domain obtained from the polydisc $\mathbb{C} \times \Delta$ by removing a closed subset $B$ whose section $B_{\tau}$ with $\tau=$ const. is always compact and reduces to a point $\{\varphi(t)\}$ when $\tau=t$ is real. Then $\varphi(t)$ is real-analytic unless $F(z, \tau)$ can be continued to the whole polydisc.

This lemma was proved by T. Ohsawa (see Kaneko [11]). Similar results were also given by Sadullaev \& Chirka [1]. These depend on the Oka-Nishino- 
Yamaguchi theory on the distribution of parabolic Riemann surfaces, which may itself be considered as belonging to the field of continuation problems. We also note a work of Kawai [4] which gave a new interpretation to Hartogs' original theorem from the viewpoint of partial differential equations.

As a converse to the above we have the following:

TheOREM N. Let $r^{0}(P)$ denote the minimal value of the dimension of smooth submanifolds in $\mathbb{R}^{n}$ which are timelike, i.e. have only non-characteristic conormals. If $C$ is a real-analytic timelike submanifold of dimension $r^{0}(P)$, we can find a real-analytic solution $u$ of $P u=0$ defined outside $C$ which has $C$ as nonremovable singularity.

There is some gap between the two theorems. Generically, Theorem N covers the interior of the region treated by Theorem L. Besides, there is one significant defect compared to Fourier analysis or the Fundamental Principle in the case of constant coefficients: the irreducible components are not dealt with well in these results. We have some remedy for this (the Fundamental Principle for operators with variable coefficients at the vertex of a cone). This idea was sketched in Kaneko [10]. In the case of a thin singularity $K$ a concrete result is given in Kaneko [8] but is not yet sufficiently well developed. See also a recent work of Uchida [1] which solved this problem for a class of operators containing the wave operator at the edge of a thick wedge.

The idea of the micro-localization of the problem of continuation of solutions is explained in our former survey Kaneko [5]. Further development on the analyticity of a minimal dimensional singularity is explained in a more recent survey Kaneko [12], [16].

We now consider how our study relates to other types of continuation problems listed at the beginning. Problems of type (1) have a different origin, coming from hyperbolicity: A solution can be continued to any hyperbolic direction (preserving the regularity of the solution) across a hypersurface. When we consider a generalization of Theorem A to non-compact $K$, we must also take into account this hyperbolicity, as is already pointed out in Malgrange [2]. A result is as follows:

TheOREM O (Kaneko [2]). Let $K$ be the part in $x_{n}<0$ of some convex compact set, and let $U$ be a neighborhood of $K$. Then the following are equivalent:

1) Every hyperfunction solution $u$ of the system $P(D) u=0$ defined in $U \backslash K$ can be continued to $U$.

2) $\operatorname{Ext}^{1}\left(\operatorname{Coker}^{t} P, \mathcal{P}\right)$ is hyperbolic in the direction $d x_{n}$, and its propagation cone $C$ satisfies

$$
\forall a \in K, \quad(\{a\}+C) \cap\left\{x_{n}<0\right\} \subset K .
$$

To our surprise, it was revealed that after having started with an apparently very different method via Fourier analysis, we came back to the hyperbolicity at the micro-local level. Thus these methods are, grosso modo, similar as regards the existence of characteristics. 
Continuation of holomorphic solutions in the complex domain is also a problem in the category of hyperbolicity. We shall not discuss this topic, but list some references: Kiselman [1], Zerner [1], Bengel [1], Bony \& Schapira [1], Tsuno [1], [2], Pallu de la Barrière [1], Presson [1], [2], Tajima [1], etc. These works are not directly related to ours. (It is too much easy to suppose that a result of continuation in the complex domain may directly imply one for real-analytic solutions.) But they have important connections with ours in the sense that they provide some of results on the propagation of micro-analyticity.

The study of removable singularities with growth condition posed on the solutions around the singularity is just a successor to the original theorem of Riemann. In this direction, studies were made to weaken the boundedness assumption or to enlarge the singularity set from isolated points to those of capacity zero etc., and for general elliptic equations, even non-linear ones. We shall not discuss these but refer to a good survey by Polking [1]. We remark here on two topics which are missing in his survey: Some results which may be classified into this category are of particular interest even to our present problem. For example, the following result of Grushin [1] rather inspired us all at the outset (see the similarity of the statement with Theorem $\mathrm{C}$ ):

THEOREM P. Let $P$ be a single linear partial differential operator with constant coefficients. The following are equivalent:

1) Every weak isolated singularity of infinitely differentiable solutions of $P(D) u=0$ is removable.

2) $P$ has no hypoelliptic irreducible factor.

Here weak singularity means that the solution can be extended as a distribution to the singularity set. For this it is sufficient that the solution is of growth at most $O\left(|x-a|^{-N}\right)$ at the singularity $a$, for the some finite $N$. Palamodov [1] generalized this result to the case of systems, replacing condition 2) by the one for $\operatorname{Ext}^{1}\left(\operatorname{Coker}^{t} P, \mathcal{P}\right)$. There are still some isolated results in this category, such as Chudov [1] etc. of which clarification from a general viewpoint would be interesting. A micro-local approach to the study of capacity arguments would also be interesting. Another topic is that for some non-linear elliptic equations isolated singularities become removable irrespective of the boundedness of the solution thanks to the non-linear effect. See e.g. Finn [1]. We expect that the significance of this work to the recent study of the one-point blowing up of solutions to non-linear equations will soon be fully appreciated, and may possibly be related to micro-local analysis.

In closing our general survey, we call readers' attention to our terminology. Our use of singularity of the solution is different from its usual use in the theory of partial differential equations: Singularity in our sense implies that the solution does not satisfy the equation at the singularity set in any reasonable sense, whereas in its usual meaning it implies that the solution satisfies the equation but only lacks the regularity under consideration. Our use is rather consistent with 
the one in classical complex analysis. For elliptic equations, there is no ambiguity between these two, because once $u$ is a solution it must be very regular. Since we mainly treat non-elliptic equations we need to avoid confusion. In our study we first look for the continuation of solutions in its weakest sense, namely as hyperfunction solutions. We then examine if we can assure the same regularity as the original solution to the continued one.

Appendix. A proof of Theorem C. In order not to finish this talk without substantial discussion, we present here a sketch of the proof for Theorem $\mathrm{C}$ in the introduction. Our method is based on Fourier analysis. It starts with Grushin's idea of representing the obstruction to the continuation via spaces of holomorphic functions on the variety of zeros of $P(\zeta)$ :

$$
N(P):=\left\{\zeta \in \mathbb{C}^{n} ; P(\zeta)=0\right\}
$$

First we give our special notation.

For an open set $U$ we let $\mathcal{A}_{P}(U)$ denote the space of real-analytic solutions of $P(D) u=0$ in $U$. Let $K \subset U$ be a pair of a convex compact set and its neighborhood. Then the quotient space

$$
\mathcal{A}_{P}(U \backslash K) / \mathcal{A}_{P}(U)
$$

represents the obstruction to continuation of real-analytic solutions from $U \backslash K$ to $U$. We also consider auxiliary spaces of hyperfunction solutions $\mathcal{B}_{P}(U)$ etc. Let $\mathcal{B}[K]$ denote the space of hyperfunctions with supports in $K$. Since $\mathcal{B}_{P}(U) \cap$ $\mathcal{B}[K]=0$, corollary to Holmgren's uniqueness theorem, we can consider $\mathcal{B}_{P}(U) \subset$ $\mathcal{B}_{P}(U \backslash K)$. Thus the quotient space $\mathcal{B}_{P}(U \backslash K) / \mathcal{B}_{P}(U)$ represents the obstruction to continuation of hyperfunction solutions. Further, since $\mathcal{B}_{P}(U) \cap \mathcal{A}(U \backslash K)=$ $\mathcal{A}_{P}(U)$, which is a kind of propagation of real analyticity, we consider $\mathcal{A}_{P}(U \backslash$ $K) / \mathcal{A}_{P}(U) \subset \mathcal{B}_{P}(U \backslash K) / \mathcal{B}_{P}(U)$. In other words, even for the continuation of realanalytic solutions it suffices to see its possibility as hyperfunction solutions. Let $u \in \mathcal{B}_{P}(U \backslash K)$. In view of the flabbiness of $\mathcal{B}$, we can extend it to a hyperfunction $[u]$ on $U$. Then $P(D)[u]$ becomes an element of $\mathcal{B}[K]$ which is well defined modulo $P(D) \mathcal{B}[K]$. This ambiguity disappears when we apply the Fourier transformation and restrict the result to the variety $N(P)$. Thus we obtain the canonical mapping, which we shall call the Grushin representation:

$$
\mathcal{B}_{P}(U \backslash K) / \mathcal{B}_{P}(U) \stackrel{\sim}{\rightarrow} \widetilde{\mathcal{B}[K]}\{N(P)\} .
$$

Here $\widetilde{\mathcal{B}[K]}\{N(P)\}$ denotes the space of global holomorphic functions on the variety $N(P)$ satisfying the same growth condition as the Fourier image of $\mathcal{B}[K]$. The latter is given by the Paley-Wiener-Ehrenpreis theorem: for any $\varepsilon>0$ there exists $C_{\varepsilon}>0$ such that

$$
|F(\zeta)| \leq C_{\varepsilon} e^{\varepsilon|\zeta|+H_{K}(\operatorname{Im} \zeta)},
$$

where $H_{K}(\eta)$ is the supporting function of $K$. The Fundamental Principle is the statement that (A.2) is an isomorphism if $P(\zeta)$ is irreducible. As a matter of fact, 
modification for the case when $P(\zeta)$ is not irreducible is easy, but we shall assume the irreducibility of $P$ for the sake of simplicity.

Now we are interested in the image of the subspace (A.1) under the mapping (A.2). Actually, it consists of functions satisfying

$$
|F(\zeta)| \leq C e^{-\delta|\zeta|} \quad \text { for }|\operatorname{Im} \zeta| \leq \delta(|\operatorname{Re} \zeta|+1)
$$

for some $\delta>0$. This estimate comes from the real-analytic regularity of the solution. In Kaneko [1] we first proved this employing differential operators of infinite order of local type, then reproved it in Kaneko [6] by means of an approximately real-analytic sequence of cut-off functions. Here we give a new proof employing Fourier hyperfunctions. This method was exploited in Kaneko [13] to treat non-compact $K$. Note that all these three techniques are considered to be substitutes for the use of cut-off functions in the $C^{\infty}$ category. Let $u \in \mathcal{A}_{P}(U \backslash K)$, and let $[u] \in \mathcal{B}(U)$ be an extension. A result in the theory of Fourier hyperfunctions assures that we can then find $v \in \mathcal{B}\left(\mathbb{R}^{n}\right)$ such that

1) $[u]-v$ is real-analytic in $U$.

2) $v$ extends holomorphically to a neighborhood of $\mathbb{R}^{n} \backslash K$ which contains a set of the form $|\operatorname{Im} z| \leq \delta|\operatorname{Re} z|-1 / \delta$ on which $v$ satisfies $|v(z)| \leq C e^{-\delta|z|}$.

A hyperfunction satisfying the condition 2) will simply be called a section of $\mathcal{O}$ on $\mathbb{D}^{n} \backslash K$. Actually, this is a sheaf on the directional compactification $\mathbb{D}^{2 n}$ of $\mathbb{C}^{n}$ whose stalks at points in $\mathbb{C}^{n}$ agree with those of $\mathcal{O}$, and those at infinity consist of the boundary values of holomorphic functions satisfying the growth condition just described in the above condition 2). The space $\mathcal{O}\left(D^{n}\right)$ of global sections of this sheaf on the "real axis" $\mathbb{D}^{n}$ just consists of functions satisfying (A.4) for some $\delta>0$. It has natural structure of (DFS)-space, and it is stable under the classical Fourier transform. The dual $\widetilde{\mathcal{Q}}\left(\mathbb{D}^{n}\right)$ is bigger than the space $\mathcal{S}^{\prime}$ and the Fourier transform can be extended to it. It is localized to a sheaf $\widetilde{\mathcal{Q}}$ on $\mathbb{D}^{n}$, which agrees with the sheaf $\mathcal{B}$ of hyperfunctions on $\mathbb{R}^{n}$. The existence of a modification $v$ as above follows from the flabbiness of the quotient sheaf $\widetilde{\mathcal{Q}} / \mathcal{O}$ on $\mathbb{D}^{n}$. We have the following convenient criterion to measure the size of the analytic singular support $K$ via the Fourier transform:

Lemma A.1 (Kawai [1]). Let $K$ be a convex compact set. For $v \in \widetilde{\mathcal{Q}}\left(\mathbb{D}^{n}\right)$, the following are equivalent:

1) $v(x) \in \underset{\approx}{\mathcal{O}}\left(\mathbb{D}^{n} \backslash K\right)$.

2) $(\mathcal{F} v)(\zeta)$ is a real-analytic function which, for any $\delta>0$, can be holomorphically extended to a conic neighborhood $|\operatorname{Im} \zeta| \leq C_{\delta}(|\operatorname{Re} \zeta|+1)$, and there it satisfies, for any $\varepsilon>0$,

$$
|(\mathcal{F} v)(\zeta)| \leq C_{\varepsilon} e^{\varepsilon|\zeta|+H_{K}(\operatorname{Im} \zeta)+\delta|\operatorname{Im} \zeta|} .
$$

Now consider $w:=P(D)[u]-P(D) v$. By the assumption $w$ belongs to $\mathcal{A}(U)$ and also to $\underset{\mathcal{O}}{\mathcal{O}}\left(\mathbb{D}^{n} \backslash K\right)$. Hence $w \in \underset{\approx}{\mathcal{O}}\left(\mathbb{D}^{n}\right)$. Because on some domain of the form 
$|\operatorname{Im} \zeta| \leq \delta(|\operatorname{Re} \zeta|+1)$ we have

$$
\mathcal{F}(P(D)[u])=P(\zeta) \mathcal{F}(v)+\mathcal{F} w=\mathcal{F} w \quad \text { for } P(\zeta)=0,
$$

we conclude that $\mathcal{F}(P(D)[u])$ is exponentially decreasing on $N(P)$ in the real direction.

The next step is to examine if such a function can exist on $N(P)$. If it is on the free space this is denied by a theorem of Carlson extending the PhragménLindelöf principle. For a general variety $N(P)$, it depends on the existence of a real point at infinity. It is rather obvious that if $N(P)$ contains no real points at infinity, then $P$ becomes elliptic, in which case (A.1) contains a non-trivial element, e.g. $E(x-a)$, where $E$ is the fundamental solution of $P$ and $a \in K$. This is equivalent to saying that the function $F(\zeta) \equiv 1$ on $N(P)$ satisfies (A.4) when $P$ is elliptic. Assume to the contrary that $N(P)$ contains a real point at infinity. After a real-linear coordinate transformation, we can assume it to be $(0,0, \ldots, 0,1)$, hence $P_{m}(0,0, \ldots, 0,1)=0$ and further $P_{m}(1,0, \ldots, 0) \neq 0$. Then we can find a function $\tau\left(\zeta^{\prime}\right)$ defined on, say,

$$
\left\{\zeta^{\prime} \in \mathbb{C}^{n-1}:\left|\zeta^{\prime \prime}\right|<\delta, \operatorname{Im} \zeta_{n}>1 / \delta\right\}
$$

where $\zeta^{\prime \prime}=\left(\zeta_{2}, \ldots, \zeta_{n-1}\right), \zeta^{\prime}=\left(\zeta^{\prime \prime}, \zeta_{n}\right)$, such that for each fixed $\zeta^{\prime \prime}, \tau\left(\zeta^{\prime}\right)$ is holomorphic in $\zeta_{n}$ and satisfies

1) $P\left(\tau\left(\zeta^{\prime}\right), \zeta^{\prime}\right)=0$

2) $\left|\tau\left(\zeta^{\prime}\right)\right| \leq M\left(\left|\zeta_{n}\right|+1\right)$

3) $\operatorname{Im} \tau\left(\zeta^{\prime}\right)=o\left(\left|\zeta_{n}\right|\right)$.

Then $G\left(\zeta_{n}\right):=F\left(\tau\left(\zeta^{\prime \prime}, \zeta_{n}\right), \zeta^{\prime \prime}, \zeta_{n}\right)$ becomes a holomorphic function of one variable satisfying the assumption of Carlson's theorem. Especially important is the fact that thanks to the condition 3) $\left|\operatorname{Re} \zeta_{n}\right|$ only appears to small order in the estimate of $G$ on substitution of $\zeta_{1}=\tau\left(\zeta^{\prime}\right)$. Hence $G\left(\zeta_{n}\right)$ vanishes identically. Since the set of points $\left(\tau\left(\zeta^{\prime}\right), \zeta^{\prime}\right)$ corresponding to (A.5) is open in the irreducible variety $N(P)$, we conclude that $F(\zeta) \equiv 0$. Namely, the solution can be continued to $U$.

\section{References}

W. Abramczuk [1] On continuation of quasi-analytic solutions of partial differential equations to compact convex sets, J. Austral. Math. Soc. 39 (1985), 306-316.

Sh. A. Akhmedov [1] On continuation of generalized solutions of differential equations defined on a neighborhood of characteristic subspaces, Dokl. Akad. Nauk SSSR 197 (1971), 255-256.

A. Andreotti [1] Complexes of Partial Differential Operators, Yale University, 1975.

E. Bedford and T. Kawai [1] Local extension of solutions of systems of linear differential equations with constant coefficients, Comm. Pure Appl. Math. 30 (1977), 235-254.

G. Bengel [1] Sur le prolongement analytique des solutions d'une équation aux dérivées partielles, C. R. Acad. Sci. Paris 273 (1971), 572-574.

J. Bochner [1] Partial differential equations and analytic continuation, Proc. Nat. Acad. Sci. U.S.A. 38 (1952), 227-230. 
J. M. Bony et P. Schapira, [1] Existence et prolongement des solutions holomorphes des équations aux dérivées partielles, Invent. Math. 17 (1972), 95-105.

[2] Solutions hyperfonctions du problème de Cauchy, in: Lecture Notes in Math. 287, Springer, 1973, 82-98.

L. A. Chudov [1] On singularities of solutions of linear partial differential equations with constant coefficients, Dokl. Akad. Nauk SSSR 125 (1959), 504-507 (in Russian).

L. Ehrenpreis [1] A new proof and extension of Hartogs' theorem, Bull. Amer. Math. Soc. 67 (1961), 507-509

[2] Fourier Analysis in Several Complex Variables, Wiley-Interscience, 1970.

R. Finn [1] Isolated singularities of solutions of non-linear partial differential equations, Trans. Amer. Math. Soc. 75 (1953), 385-403.

E. A. Gorin and V. V. Grushin [1] On some local theorem for partial differential equations with constant coefficients, Trudy Moskov. Mat. Obshch. 14 (1965), 200-210 (in Russian).

V. V. Grushin [1] On the Q-hypoelliptic equations, Mat. Sb. 57 (1962), 233-240 (in Russian).

[2] On solutions with isolated singularities for partial differential equations with constant coefficients, Trudy Moskov. Mat. Obshch. 15 (1966), 262-278 (in Russian).

F. Hartogs [1] Einige Folgerungen aus der Cauchyschen Integralformel bei Funktionen mehrerer Veränderlichen, Sitzb. Münchener Acad. 36 (1906), 223-241.

[2] Über die aus den singulären Stellen einer analytischen Funktion mehrerer Veränderlichen bestehenden Gebilde, Acta Math. 32 (1908), 57-79.

R. Harvey and J. Polking [1] Removable singularities of solutions of linear partial differential equations, ibid. 125 (1970), 39-56.

L. Hörmander [1] On the existence of real analytic solutions of partial differential equations with constant coefficients, Invent. Math. 21 (1973), 151-182.

F. John [1] Plane Waves and Spherical Means, Interscience, 1955.

A. Kaneko [1] On continuation of regular solutions of partial differential equations to compact convex sets, J. Fac. Sci. Univ. Tokyo Sect. 1 A 17 (1970), 567-580; II, ibid. 18 (1972) $415-433$.

[2] On continuation of regular solutions of partial differential equations with constant coefficients, J. Math. Soc. Japan 26 (1974), 92-123.

[3] Note on continuation of real analytic solutions of partial differential equations with constant coefficients, Proc. Japan Acad. 51 (1975), 262-264.

[4] Singular spectrum of boundary values of solutions of partial differential equations with real analytic coefficients, Sci. Pap. Coll. Gen. Educ. Univ. Tokyo 25 (1975), 59-68.

[5] On continuation of regular solutions of linear partial differential equations, Publ. RIMS Kyoto Univ. 12 Suppl. (1977), 113-121.

[6] Prolongement des solutions régulières de l'équation aux dérivées partielles à coefficients constants, Séminaire Goulaouic-Schwartz, 1976/7, Exposé no 18.

[7] Estimation of singular spectrum of boundary values for some semi-hyperbolic operators, J. Fac. Sci. Univ. Tokyo Sec. 1A 27 (1980), 401-461.

[8] On continuation of real analytic solutions of linear partial differential equations, Astérisque 89-90 (1981), 11-44.

[9] On the propagation of micro-analyticity along the boundary, J. Fac. Sci. Univ. Tokyo Sect. 1A 29 (1982), 319-352.

[10] Continuation of real analytic solutions to convex conical singularities, Sûrikaisekikenkyûsho Kôkyûroku 592 (1986), 149-172 (in Japanese).

[11] On the analyticity of the locus of singularity of real analytic solutions with minimal dimension, Nagoya Math. J. 104 (1986), 63-84.

[12] Nishino-Yamaguchi theory and its application to the theory hyperfunctions, in: Proc.

7th Daewoo Workshop on Pure Math., Seoul, 1987, 241-261.

[13] On Hartogs type continuation theorem for regular solutions of linear partial differential 
equations with constant coefficients, J. Fac. Sci. Univ. Tokyo 35 (1988), 1-26.

[14] Hartogs type extension theorem of real analytic solutions of linear partial differential equations with constant coefficients, in: Advances in the Theory of Fréchet Spaces, Kluwer, 1989, 63-72.

[15] Introduction to Hyperfunctions, Kluwer, Tokyo 1988.

[16] Analyticité du lieu de singularité de dimension minimale d'une solution analytique réelle, in: Geometrical and Algebraical Aspects in Several Complex Variables, Editel, Rende, 1991, $155-167$.

K. Kataoka [1] Micro-local theory of boundary value problems II, J. Fac. Sci. Univ. Tokyo Sect. 1A 28 (1981), 31-56.

T. Kawai [1] On the theory of Fourier hyperfunctions and its applications to partial differential equations with constant coefficients, ibid. 17 (1970), 467-517.

[2] Removable singularities of solutions of systems of linear differential equations, Bull. Amer. Math. Soc. 81 (1975), 461-463.

[3] Extension of solutions of systems of linear differential equations, Publ. RIMS Kyoto Univ. 12 (1976), 215-227.

[4] A differential equation theoretic interpretation of a geometric result of Hartogs, Proc. Amer. Math. Soc. 98 (1986), 222-224.

C. O. Kiselman [1] Prolongement des solutions d'une équation aux dérivées partielles à coefficients constants, Bull. Soc. Math. France 97 (1969), 329-356.

H. Komatsu [1] Relative cohomology of sheaves of solutions of differential equations, Séminaire Lions-Schwartz, 1966-67 (reprinted in: Lecture Notes in Math. 287, Springer, 1973, 192261).

H. Komatsu and T. Kawai [1] Boundary values of hyperfunction solutions of linear partial differential equations, Publ. RIMS Kyoto Univ. 7 (1971), 95-104.

W. Littman [1] Polar sets and removable singularities of partial differential equations, Ark. Mat. 7 (1967), 1-9.

B. Malgrange, [1] Sur la propagation de la régularité des solutions des équations à coefficients constants, Bull. Math. Soc. Roumanie 3 (1959), 433-440. [2] Sur les systèmes différentiels à coefficients constants, Séminaire Leray, 1961-62.

A. Meril et D. C. Struppa [1] Phénomène de Hartogs et équations de convolution, Séminaire Lelong-Skoda, 1985-86, Lecture Notes in Math. 1295, Springer, 146-156.

T. Ôaku [1] Boundary value problems for systems of linear partial differential equations and propagation of micro-analyticity, J. Fac. Sci. Univ. Tokyo Sect. 1A 33 (1986), 175-232.

[2] Removable singularities of solutions of linear partial differential equations-systems and Fuchsian equations, ibid., 403-428.

K. Oka [1] Note sur les familles de fonctions analytiques multiformes etc., J. Hiroshima Univ. 4 (1934), 93-98.

V. P. Palamodov [1] Linear Differential Equations with Constant Coefficients, Nauka, Moscow 1967 (in Russian; English translation 1970 from Springer; Japanese translation 1972-73 from Yoshioka, Kyoto).

Ph. Pallu de la Barrière [1] Existence et prolongement des solutions holomorphes des équations aux dérivées partielles, C. R. Acad. Sci. Paris 279 (1974), 947-949.

J. Presson [1] Local analytic continuation of holomorphic solutions of partial differential equations, Ann. Mat. Pura Appl. 112 (1977), 193-204.

[2] On the analytic continuation of holomorphic solutions of partial differential equations, Ark. Mat. 19 (1981), 177-191.

J. C. Polking [1] A survey of removable singularities, in: Seminar on Non-linear Partial Differential Equations, S. S. Chern (ed.), Springer, 1984, 261-292.

B. Riemann [1] Grundlagen für eine allgemeine Theorie der Functionen einer veränderlichen complexen Grösse, Gesam. Math. Werke, 3-48. 
A. Sadullaev and E. M. Chirka [1] On continuation of functions with polar singularities, Mat. Sb. 132 (1987), 383-390.

P. Schapira [1] Propagation au bord et reflexion des singularités analytiques des équations aux dérivées partielles II, Séminaire Goulaouic-Schwartz 1976-77, exposé no. 9.

[2] Propagation at the boundary of analytic singularities, in: Proceedings NATO Symposium Maratea, Reidel, 1980, 185-212.

[3] Propagation of analytic singularities up to non smooth boundaries, in: Colloque E.D.P. Saint-Jean de Monts, 1987.

F. Severi [1] Una proprietà fondamentale dei campi di olomorfismo di una funzione analitica di una variabile reale e di una variabile complessa, Rend. R. Accad. Lincei 15 (1932), 487-490.

D. Struppa [1] The first eighty years of Hartogs theorem, in: Seminari di Geometria 1987-88, Univ. di Bologna, 127-209.

S. Tajima [1] Analyse microlocale sur les variétés de Cauchy-Riemann et le problème du prolongement des solutions holomorphes des équations aux dérivées partielles, Publ. RIMS Kyoto Univ. 18 (1982), 911-945.

Y. Tsuno [1] On the prolongation of local holomorphic solutions of partial differential equations, J. Math. Soc. Japan 26 (1974), 523-548.

[2] On the prolongation of local holomorphic solutions of non-linear partial differential equations, ibid. 27 (1975), 454-466.

[3] Analytic continuation of holomorphic solutions of partial differential equations, Sûrikaiseki-Kenkyûsho Kôkyûroku 281 (1976), 120-162 (in Japanese).

M. Uchida [1] Continuation of analytic solutions to linear partial differential equations up to convex conical singularities, preprint.

M. Uchida and G. Zampieri [1] Second microlocalization at the boundary and microhyperbolicity, Publ. RIMS Kyoto Univ. 26 (1990), 205-219.

G. Zampieri [1] Propagation of microanalyticity at the boundary for solutions of linear differential equations, J. Fac. Sci. Univ. Tokyo Sect. 1A 33 (1986), 429-439.

M. Zerner [1] Domaine d'holomorphie des fonctions vérifiant une équation aux dérivées partielles, C. R. Acad. Sci. Paris 272 (1971), 1646-1648. 